\title{
ALGAS MARINHAS BENTÔNICAS DA RESERVA BIOLÓGICA ESTADUAL DA PRAIA DO SUL, ILHA GRANDE, ANGRA DOS REIS, RIO DE JANEIRO, BRASIL
}

\author{
Lísia Mônica de Souza Gestinari ${ }^{1}$ \\ Cristina Aparecida Gomes Nassar \\ Patrícia Valéria dos Santos Arantes
}

Recebido em 14/11/94. Aceito em 11/3/98

\begin{abstract}
RESUMO - (Algas marinhas bentônicas da Reserva Biológica Estadual da Praia do Sul, tha Grande, Angra dos Reis, Rio de Janeiro, Brasil). Na Reserva foram encontradas 121 espécies, sendo 27 de Chlorophyceac, 21 de Phaeophyceae e 73 de Rhodophyceae. Dentre estas espécies, 38 são novas referências para a ilha, sendo que Cladophora catenata (L.) Kütz. é pela primeira vez citada para o litoral do Rio de Janeiro. Nào foram encontradas espécies endêmicas e nem flora especifica de região insular. A comparação da flora da Reserva com a de regiões vizinhas indica alta percentagem de espécies em comum entre elas.
\end{abstract}

Palavras-chave: algas marinhas bentônicas, taxonomia, Unidade de Conservação, Ilha Grande

ABSTRACT - (Benthic marine algae of the State Biological Reserve of Praia do Sul, llha Grande, Angra dos Reis, Rio de Janeiro, Brazil). A survey in the Reserve showed 27 species of Chlorophyceae, 21 of Phaeophyceae and 73 of Rhodophyceae. Among the recorded species, 38 are new references to the Island and Cladophora catenata (L.) Kütz. is for the first time quoted to the Rio de Janeiro coast. Neither endemic species nor specific flora from insular area were found in the Reserve. A comparison between the flora of the Reserve and those of neighboring areas, showed a high percentage of similarity.

Key words: benthic marine algae, taxonomy, conservation unit, Ilha Grande

\section{Introdução}

A Ilha Grande está localizada ao sul do Estado do Rio de Janeiro $\left(23^{\circ} 4^{\prime} 23^{\circ} 13^{\prime} \mathrm{S}\right.$ e $\left.44^{\circ} 5^{\prime} 44^{\circ} 22^{\prime} \mathrm{W}\right)$, próxima à Baía de Sepetiba e à Baía de Angra dos Reis.

Criada em 2/12/81 pelo Decreto Estadual n. ${ }^{\circ} 4972$, a Reserva Biológica Estadual da Praia do Sul (R.B.E.P.S.) veio assegurar a conservação da integridade e manejo dos ecossistemas ao sul da Ilha Grande (Maciel et al. 1984). A preservação dos diversos ecossistemas ali encontrados, altamente ricos em espécies da flora e da fauna, foram

\footnotetext{
Laboratório Integrado de Ficologia, Departamento de Botânica, Instituto de Biologia, CCS, Universidade Federal do Rio de Janeiro, Ilha do Fundão, CEP 21941-900, Rio de Janeiro, RJ, Brasil
} 
possíveis devido a dificuldade de acesso, tanto por mar quanto por terra (Maciel et al. 1981).

Diversos trabalhos foram realizados na referida reserva (Maciel et al. 1981; Maciel et al. 1984), porém a maioria deles está relacionado com a flora e fauna terrestres. A presente contribuição é relevante devido a inexistência de citações de algas marinhas bentônicas para esta Unidade de Conservação.

O principal objetivo foi realizar o levantamento das espécies que ocorrem na Reserva, fornecendo assim subsídios para a preservação do seu patrimônio florístico. Além deste aspecto, o estudo visa ainda contribuir para o melhor conhecimento da distribuição das espécies ao longo da costa do Estado do Rio de Janeiro e do Brasil.

\section{Material e métodos}

O material analisado corresponde a quatro coletas realizadas em: 24/IX/1988; 4-5/VII/1989; 10-12/II/1992 e 28-30/VII/1992.

As coletas foram feitas ao acaso, com o auxílio de espátulas ao longo dos costões rochosos e no manguezal da Reserva (Fig. 1): estação 1 - Praia do Sul, no costão da Ilhota do Leste, voltado para esta praia; estação 2 - Praia do Leste, no costão da Ilhota do Leste, voltado para esta praia; estação 3 - Praia do Aventureiro; estação 4 - Pedra do Demo. Excetuando a Praia do Aventureiro, as demais estações apresentaram alto grau de exposição às ondas; estação 5 - Borda do manguezal do Rio Ariró, que desemboca na Praia do Leste. Nesta estação o material foi coletado sobre as raízes de Rhizophora sp. e Avicennia sp.

$\mathrm{Na}$ listagem das espécies (Tab. 1) um asterisco (*) indica que a espécie é citação nova para a Ilha Grande, e dois asteriscos (**) indicam citação nova para o litoral do Rio de Janeiro.

Neste trabalho adotou-se a revisão nomenclatural de gêneros e espécies proposta por Wynne (1986), com exceção das Phaeophyceae, onde para algumas espécies de Ectocarpales, seguiu-se Széchy \& Cordeiro-Marino (1991); para as espécies Leptonematella fasciculata e Dictyota pardalis utilizou-se Yoneshigue (1985) e, para Padina vickersiae, Széchy (1986). Para a identificação das espécies de Corallinaceae (Rhodophyceae) foi utilizado o estudo de Piqué (1984).

\section{Resultados}

Foi encontrado o total de 56 gêneros e 121 espécies na área de estudo (Tab. 1), compreendendo 27 espécies da Classe Chlorophyceae (22,3\%), 21 da Classe Phaeophyceae $(17,3 \%)$, e 73 da Classe Rhodophyceae $(60,4 \%)$. Dentre as espécies encontradas, 38 são citações novas para a Ilha Grande. A Ordem Cladophorales foi a mais representativa entre as Chlorophyceae, com 11 espécies. Entre as Phaeophyceae, a Ordem Ectocarpales com sete espécies foi a mais significativa e, nas Rhodophyceae, as Ceramiales ocorreram de forma mais expressiva, com 43 espécies. Dentre as espécies identificadas, Polysiphonia subtilissima foi a única que ocorreu em todos os pontos de coleta. Cladophora catenata é pela primeira vez citada para o litoral do Estado do Rio de Janeiro. 


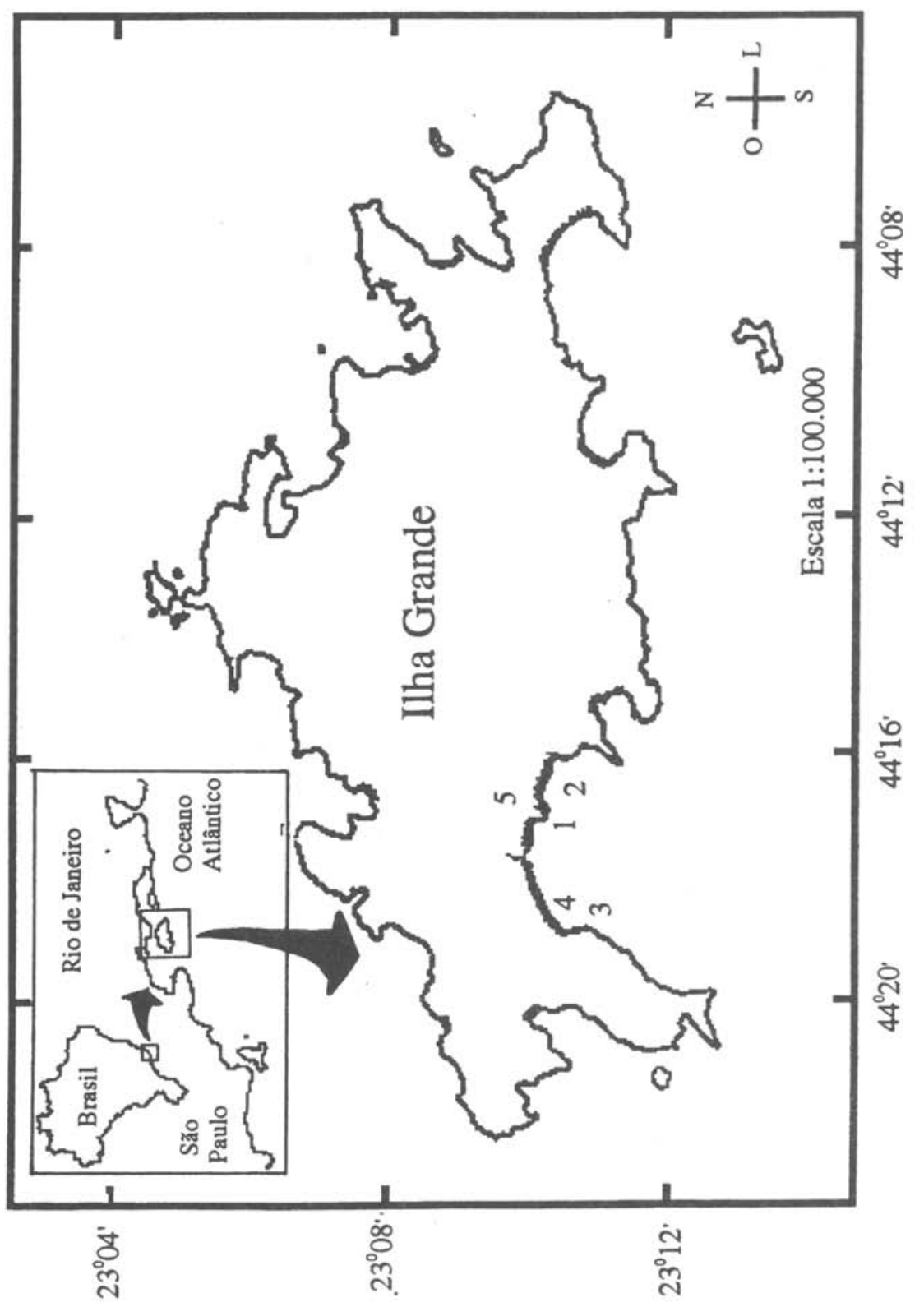

Figura 1. Ilha Grande. Mapa da região estudada. (1) Praia do Sul. (2) Praia do Leste. (3) Praia do Aventureiro. (4) Pedra do Demo. (5) Borda do manguezal do Rio Ariró. 
Tabela 1. Listagem dos táxons encontrados nas cinco estações de coleta (+ presença; - ausência; * citação nova para a Illha Grande; ** Citação nova para o Rio de Janeiro)

\begin{tabular}{|c|c|c|c|c|c|}
\hline \multirow[t]{2}{*}{ TAXON } & \multicolumn{5}{|c|}{ ESTAÇĀO DE COLETA } \\
\hline & 1 & 2 & 3 & 4 & 5 \\
\hline \multicolumn{6}{|l|}{$\overline{\text { CHLOROPHYTA }}$} \\
\hline \multicolumn{6}{|l|}{ ULVALES } \\
\hline \multicolumn{6}{|l|}{ ULVACEAE } \\
\hline *Enteromorpha clathrata (Roth) Greville & - & - & - & - & + \\
\hline${ }^{*}$ E. compressa $(\mathrm{L}$.) Greville & - & + & - & - & - \\
\hline E. flexuosa (Wulfen ex Roth) J. Agardh & + & + & + & + & - \\
\hline${ }^{*} E$. linza (L.) J. Agardh & - & - & + & - & - \\
\hline Ulva fasciata Delile & + & + & + & + & - \\
\hline U. lactuca $\mathrm{L}$. & + & - & + & + & - \\
\hline Ulvaria oxysperma (Kützing) Bliding & - & + & - & - & - \\
\hline \multicolumn{6}{|l|}{ SIPHONOCLADALES } \\
\hline \multicolumn{6}{|l|}{ SIPHONOCLADACEAE } \\
\hline Cladophoropsis membranacea (C.Agardh) Boergesen & + & + & - & - & - \\
\hline \multicolumn{6}{|l|}{ CLADOPHORALES } \\
\hline \multicolumn{6}{|l|}{ CLADOPHORACEAE } \\
\hline Chaetomorpha aerea (Dillwyn) Kützing & + & - & + & - & - \\
\hline C. antennina (Bory) Kützing & + & + & + & + & - \\
\hline${ }^{*}$ C. brachygona Harvey & - & - & - & + & - \\
\hline **Cladophora catenata (L.) Kützing & + & - & - & - & - \\
\hline${ }^{*}$ C. coelothrix Kützing & - & + & - & - & - \\
\hline C. corallicola Boergesen & - & + & + & - & - \\
\hline${ }^{*}$ C. montagneana Kützing & - & + & - & - & - \\
\hline C. prolifera (Roth) Kützing & - & + & - & - & - \\
\hline${ }^{*}$ C. rupestris (L.) van den Hoek & - & - & + & - & - \\
\hline C. vagabunda (L.) van den Hoek & + & + & - & - & - \\
\hline * Rhizoclonium africanum Kützing & - & - & - & - & + \\
\hline \multicolumn{6}{|l|}{ CAULERPALES } \\
\hline \multicolumn{6}{|l|}{ BRYOPSIDACEAE } \\
\hline Bryopsis pennata Lamouroux & + & + & + & + & - \\
\hline *B. plumosa (Hudson) C.Agardh & - & - & + & - & - \\
\hline \multicolumn{6}{|l|}{ CODIACEAE } \\
\hline Codium decorticatum (Woodward) Howe & + & - & - & - & - \\
\hline C. intertextum Collins \& Harvey & + & + & + & + & - \\
\hline C. taylorii Silva & + & + & + & - & - \\
\hline \multicolumn{6}{|l|}{ CAULERPACEAE } \\
\hline *Caulerpa ambigua Okamura & + & - & + & - & - \\
\hline C. fastigiata Montagne & - & + & + & - & - \\
\hline C. racemosa (Forsskal) J. Agardh & + & - & + & - & - \\
\hline \multicolumn{6}{|l|}{ PHAEOPHYTA } \\
\hline \multicolumn{6}{|l|}{ ECTOCARPALES } \\
\hline \multicolumn{6}{|l|}{ ECTOCARPACEAE } \\
\hline Bachelotia antillarum (Grunow) Gerloff & - & + & - & - & - \\
\hline Feldmannia simplex (Crouan \& Crouan) Hamel & - & - & + & - & - \\
\hline Hincksia breviarticulata (J. Agardh) Silva & + & + & + & + & - \\
\hline H. irregularis (Kützing) Széchy \& Cordeiro-Marino & + & + & + & + & - \\
\hline H. mitchelliae (Harvey) Silva & - & - & + & + & - \\
\hline *Leptonematella fasciculata (Reinke) Silva & - & - & + & - & - \\
\hline \multicolumn{6}{|l|}{ RALFSIACEAE } \\
\hline Ralfsia expansa (J. Agardh) J. Agardh & + & + & + & - & - \\
\hline \multicolumn{6}{|l|}{ CHORDARIALES } \\
\hline CHORDARIACEAE & & & & & \\
\hline Levringia brasiliensis (Montagne) Joly & - & - & + & - & - \\
\hline
\end{tabular}


Tabela 1. Listagem dos táxons encontrados nas cinco estações de coleta (+ presença; - ausência; * citação nova para a Ilha Grande; ** Citação nova para o Rio de Janeiro) - Continuação

\begin{tabular}{|c|c|c|c|c|c|}
\hline \multirow[t]{2}{*}{ TAXXN } & \multicolumn{5}{|c|}{ ESTAÇÁO DE COLETA } \\
\hline & 1 & 2 & 3 & 4 & 5 \\
\hline \multicolumn{6}{|l|}{ SCYTOSIPHONALES } \\
\hline \multicolumn{6}{|l|}{ CHNOOSPORACEAE } \\
\hline \multirow{2}{*}{\multicolumn{6}{|c|}{ SCYTOSIPHONACEAE }} \\
\hline & & & & & \\
\hline Colpomenia sinuosa (Roth) Derbès \& Solier & + & + & + & + & - \\
\hline \multicolumn{6}{|l|}{ SPHACELARIALES } \\
\hline \multicolumn{6}{|l|}{ SPHACELARIACEAE } \\
\hline Sphacelaria brachygonia Montagne & + & + & + & + & - \\
\hline S. rigidula Kützing & - & - & + & - & - \\
\hline S. tribuloides Meneghini & + & - & + & + & - \\
\hline \multicolumn{6}{|l|}{ DICTYOTALES } \\
\hline \multicolumn{6}{|l|}{ DICTYOTACEAE } \\
\hline Dictyopteris delicatula Lamouroux & + & + & + & + & - \\
\hline D. cervicornis Kützing & + & - & + & + & - \\
\hline D. ciliolata Kützing & - & + & + & + & - \\
\hline *D. pardalis Kützing & + & - & - & - & - \\
\hline Padina gymnospora (Kützing) Sonder & + & + & + & + & - \\
\hline *P. vickersiae Hoyt ex Howe & - & + & + & + & - \\
\hline \multicolumn{6}{|l|}{ FUCALES } \\
\hline \multicolumn{6}{|l|}{ SARGASSACEAE } \\
\hline \multicolumn{6}{|l|}{ Sargassum cymosum C. Agardh var. nanum } \\
\hline Paula \& Oliveira-Filho & + & + & + & + & - \\
\hline \multicolumn{6}{|l|}{ S. vulgare C. Agardh var. foliosissimum } \\
\hline (Lamouroux) C. Agardh & - & - & + & - & - \\
\hline \multicolumn{6}{|l|}{ RHODOPHYTA } \\
\hline \multicolumn{6}{|l|}{ COMPSOPOGONALES } \\
\hline \multicolumn{6}{|l|}{ ERYTHROPELTIDACEAE } \\
\hline Erythrotrichia carnea (Dillwyn) J. Agardh & - & + & + & - & - \\
\hline \multicolumn{6}{|l|}{ BANGIALE } \\
\hline \multicolumn{6}{|l|}{ BANGIACEAE } \\
\hline *Bangia atropurpurea (Roth) C. Agardh & - & + & - & - & - \\
\hline $\begin{array}{l}\text { Porphyra acanthophora Oliveira \& Coll var. acanthophora } \\
\text { NEMALIALES }\end{array}$ & + & + & + & + & - \\
\hline \multicolumn{6}{|l|}{ GALAXAURACEAE } \\
\hline Galaxaura marginata (Ellis \& Solander) Lamouroux & - & - & + & - & - \\
\hline \multicolumn{6}{|l|}{ GELIDIALES } \\
\hline \multicolumn{6}{|l|}{ GELIDIACEAE } \\
\hline Gelidium floridanum Taylor & - & - & + & - & - \\
\hline G. pusillum (Stackhouse) Le Jolis & + & + & + & - & - \\
\hline $\begin{array}{l}\text { Pterocladia capillacea (Gmelin) Bornet \& Thuret } \\
\text { GELIDIELLACEAE }\end{array}$ & + & + & + & - & - \\
\hline Gelidiella trinitatensis Taylor & + & - & - & - & - \\
\hline BONNEMAISONIALES & & & & & \\
\hline BONNEMAISONIACEAE & & & & & \\
\hline Asparagopsis taxiformis (Delile) Trevisan & + & + & + & + & - \\
\hline CORALLINALES & & & & & \\
\hline CORALLINACEAE & & & & & \\
\hline *Amphiroa beauvoisii Lamouroux & + & + & + & + & - \\
\hline *A. brasiliana Decaisne & - & - & + & - & - \\
\hline Arthrocardia gardneri Manza & - & - & + & - & - \\
\hline *A. stephensonii Manza & + & + & + & + & - \\
\hline
\end{tabular}


Tabela 1. Listagem dos táxons encontrados nas cinco estações de coleta (+ presença; - ausência; * citação nova para a llha Grande; ** Citação nova para o Rio de Janeiro) - Continuação

TÁXON ESTAÇÃO DE COLETA

* Haliptilon cubense (Montagne ex Kützing)

Garbary \& Johansen

Jania adhaerens Lamouroux

J. prolifera Joly

J. rubens (L.) Lamouroux

GIGARTINALES

HYPNEACEAE

Hypnea cervicornis J. Agardh

H. musciformis (Wulfen in Jacquin) Lamouroux

H. spinella (C. Agardh) Kützing

GRACILARIACEAE

Gelidiopsis gracilis (Kützing) Vickers

G. planicaulis (Taylor) Taylor

PHYLLOPHORACEAE

Gymnogongrus griffthsiae (Turner) Martius

GIGARTINACEAE

Gigartina acicularis (Roth) Lamouroux

* G. teedii (Roth) Lamouroux

CRYPTONEMIALES

HALYMENIACEAE

Grateloupia filicina (Lamouroux) C.Agardh

RHODYMENIALES

CHAMPIACEAE

Champia parvula (C. Agardh) Harvey

${ }^{*}$ C. salicornioides Harvey

C. vieillardii Kützing

RHODYMENIACEAE

* Rhodymenia pseudopalmata (Lamouroux) Silva

CERAMIALES

CERAMIACEAE

Callithamnion byssoides Arnott ex Harvey in Hooker

C. felipponei Howe

C. uruguayense Taylor

Centroceras clavulatum (C.Agardh in Kunt)

Montagne in Durieu de Maisonneuve

Ceramium brasiliense Joly

C. brevizonatum Petersen

C. codii (Richards) Mazoyer

C. comptum Boergesen

C. dawsoni Joly

C. flaccidum (Kützing) Ardisson

C. luetzelburgii Schmidt

C. strictum Harvey

C. tenerrimum (Martens) Okamura

C. tenuissimum (Roth) Areschoug

C. vagabunda Dawson

Wrangelia argus (Montagne) Montagne

DASYACEAE

Dasya brasiliensis Oliveira \& Braga

D. corymbifera J. Agardh

Heterosiphonia crispella (C. Agardh) Wynne

+
+
+
+

2 3 4

$\begin{array}{lllll}+ & + & + & + & - \\ + & + & + & + & - \\ + & + & + & + & - \\ + & + & + & - & - \\ - & - & + & + & - \\ + & - & + & + & - \\ + & - & + & - & - \\ - & - & + & -\end{array}$


Tabela 1. Listagem dos táxons encontrados nas cinco estações de coleta (+ presença; - ausência; * citação nova para a Ilha Grande; ** Citação nova para o Rio de Janeiro) - Continuação TÁXON ESTACุÃO DE COLETA

\begin{tabular}{|c|c|c|c|c|c|}
\hline & 1 & 2 & 3 & 4 & \\
\hline RHODOMELACEAE & & & & & \\
\hline Bostrychia binderi Harvey & + & - & + & - & \\
\hline Bostrychia calliptera (Montagne) Montagne & - & - & - & - & + \\
\hline B. radicans $\mathrm{f}$. moniliformis Post & - & - & + & - & \\
\hline B. radicans f. radicans (Montagne) Montagne in Kützing & - & + & + & - & \\
\hline B. tenella (Vahl) J. Agardh & + & - & + & - & \\
\hline Bryocladia cuspidata (J. Agardh) De Toni & + & + & + & - & \\
\hline B. thyrsigera (J. Agardh) Schmitz in Falkenberg & + & + & - & + & - \\
\hline Chondria atropurpurea Harvey & - & - & + & + & \\
\hline Herposiphonia secunda (C.Agardh) Falkenberg f. secunda & + & + & + & - & - \\
\hline H. secunda $\mathrm{f}$ tenella (C. Agardh) Wynne & + & + & + & + & . \\
\hline Laurencia arbuscula Sonder & + & + & + & + & \\
\hline L. flagellifera J. Agardh & - & - & + & - & \\
\hline L. implicata J. Agardh & + & - & - & - & \\
\hline L. oliveirana Yoneshigue & - & - & - & + & \\
\hline L. papilosa (C. Agardh) Greville & - & - & + & - & \\
\hline Ophidocladus simpliciusculus (Crouan \& Crouan) Falkenberg & + & + & - & + & \\
\hline Polysiphonia denudata (Dillwyn) Greville ex Harvey in Hooker & - & - & + & - & \\
\hline Polysiphonia ferulacea Suhr ex J. Agardh & - & + & - & - & \\
\hline P. havanensis Montagne & - & + & - & - & \\
\hline P. howei Hollenberg in Taylor & - & + & + & - & \\
\hline P. subtilissima Montagne & + & + & + & + & \\
\hline P. tepida Rollenberg & + & + & + & + & \\
\hline Pterosiphonia parasitica (Hudson) Falkenberg & - & + & - & - & \\
\hline P. pennata (C. Agardh) Falkenberg & - & + & + & - & \\
\hline
\end{tabular}

Cladophora catenata (L.) Kützing, Phyc. Gen.: 271. 1843.

Basiônimo: Conferva catenata L., Sp. Pl.: 1166. 1753.

(Fig. 2-5)

Plantas verde-escuro formando tapetes frouxamente compactados com cerca de $2,0 \mathrm{~cm}$ alt. Ramificação unilateral a irregular, com filamentos rigidos, geralmente encurvados, formando emaranhados. Fixação através de inúmeros filamentos rizoidais que partem das células basais e das células próximas ao eixo. Eixo principal com $100 \mu \mathrm{m}$ diâm. próximo ao ápice e $125-175 \mu \mathrm{m}$ diâm. próximo à base. Célula apical com $75-100 \mu \mathrm{m}$ diâm. e 250-275 $\mu \mathrm{m}$ de compr. Presença de hifas de fungos na parede celular. Descrição detalhada da espécie, bem como dados sobre sua ecologia, reprodução, posição sistemática e distribuição geográfica pode ser encontrada em Hoek (1982).

\section{Discussão}

Com base nos 121 táxons identificados, a região estudada pode ser considerada rica em espécies, sobretudo pelo pequeno trecho litorâneo da Reserva. Isto fica evidenciado quando se compara o número de espécies encontradas no presente estudo com regiōes vizinhas. Pedrini (1980), em estudo sobre a Baia de Sepetiba, localizada 

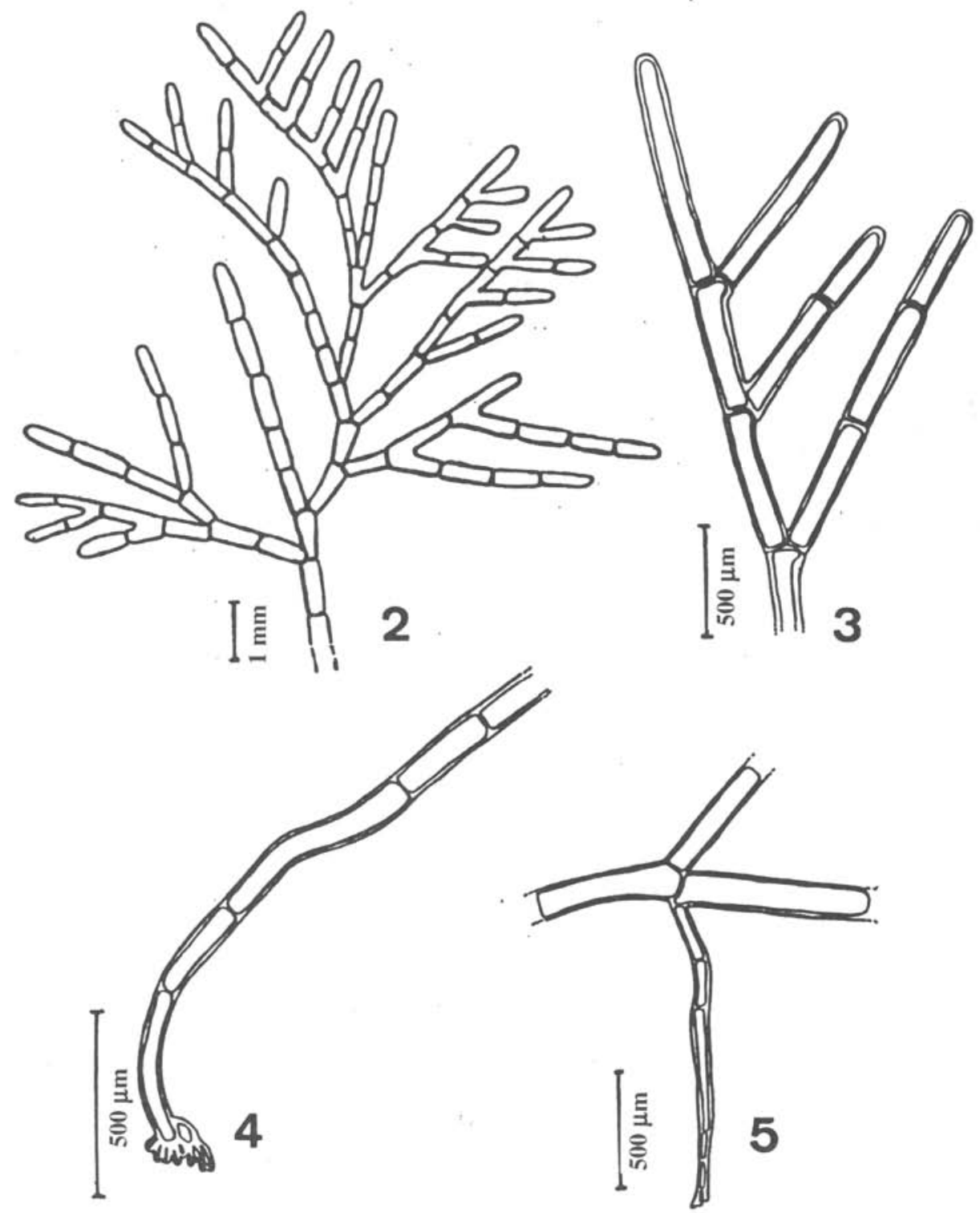

Figuras 2-5. Aspectos morfológicos de Cladophora catenata. 2. Aspecto geral do talo. 3. detalhe do ramo apical. 4. filamento basal com rizoides. 5. rizóide partindo de célula lateral. 
a leste da Ilha Grande, citou 180 espécies de macroalgas coletadas em diversos pontos, inclusive em algumas ilhas. Figueiredo (1989), estudando a flora do Municipio de Paraty, situado no continente a oeste da Ilha Grande, identificou 121 espécies. No levantamento de Falcão et al. (1992) realizado nos litorais nordeste e sudeste da ilha foi determinada a ocorrência de 128 espécies de algas. Em todos estes estudos também foi observado o maior número de espécies ocorrendo na Ordem Ceramiales (Rhodophyceae). Yoneshigue (1985), estudando a região influenciada pelas águas frias da ressurgência de Cabo Frio, igualmente demonstrou que a Ordem Ceramiales foi a que apresentou o maior número de espécies.

Em relação às Phaeophyceae, as ordens Dictyotales e Ectocarpales foram as mais numerosas e, dentre as Chlorophyceae, as ordens Cladophorales e Caulerpales foram as mais representativas, o que confere com estudos das regiões vizinhas. O predominio destas ordens demonstra bem a afinidade tropical das espécies encontradas (Diaz-Piferrer 1969).

Considerando-se o número de espécies observadas, a área estudada não se encontra tão degradada quando comparada a outros locais, como a Baía de Guanabara (107 espécies) estudada por Yoneshigue-Braga (1971; 1972a; 1972b).

A flora da Reserva apresentou alto percentual de espécies em comum com os litorais nordeste e sudeste da Ilha (49,4\%), estudados por Falcão et al. (1992). É provável que tal semelhança ocorra em função da inexistência de barreiras geográficas e térmicas na região (Signorini 1980a; 1980b; Ikeda \& Stevenson 1980), o que propicia a ocorrência da flora ficológica continua ao longo do litoral da Ilha Grande. Esse fato fica evidenciado nos estudos de Pedrini (1980) e Figueiredo (1989) que trabalharam em áreas mais distantes e ainda assim, a percentagem de espécies em comum com a Reserva foi de $47,23 \%$ e $42,35 \%$, respectivamente. A semelhança da flora, aliada a inexistência de espécies endêmicas, sugerem que algas marinhas bentônicas da Ilha Grande e, especificamente da R.B.E.P.S., podem ser consideradas como uma extensão da flora do continente e arredores.

Polysiphonia subtilissima foi a espécie que ocorreu em todas as estações de coleta, mostrando assim o seu caráter eurialino (Coutinho 1982).

Cladophora catenata é pela primeira vez citada no litoral do Rio de Janeiro. No entanto, deve-se ressaltar que esta espécie possivelmente ocorra em outros pontos do litoral deste Estado, não tendo ainda sido citada em função das dificuldades na identificação deste gênero. Esta espécie foi mencionada anteriormente por Kanagawa (1984) para o litoral da Paraiba e por Santos (1983), para o litoral de Santa Catarina.

$\mathrm{O}$ estudo da flora de algas marinhas bentônicas é de suma importância para a preservação e manejo da R.B.E.P.S. A condição insular da área estudada torna-a importante centro de dispersão de propágulos, para uma possivel recolonização de áreas continentais degradadas.

\section{Agradecimentos}

Ao professor Gilberto Mitchell, pela concessão do espaço, material e bibliografia necessários para o desenvolvimento do trabalho. À Dra. Yocie Yoneshigue-Valentin pelo auxilio na identificação de Cladophora catenata e Leptonematella fasciculata e às biólogas Cristina Falcão e Maria Cristina Maurat pela amizade e apoio nas horas mais dificeis. 


\section{Referências bibliográficas}

Coutinho, R. 1982. Taxonomia, distribuição e crescimento sazonal, reprodução e biomassa das algas bentônicas do Estuário da Lagoa dos Patos (RS). Universidade do Rio Grande do Sul, Porto Alegre. Dissertação de Mestrado.

Diaz-Piferrer, M. 1969. Distribution of the marine benthic flora of the Caribbean Sea. Caribbean Journal of Science 9(3-4): 151-177

Falcão, C.; Maurat, M.C.S.; Nassar, C.A.G.; Széchy, M.T.M. \& Mitchell, G.J.P. 1992. Benthic marine flora of the northeastern and southeastern coast of Ilha Grande, Rio de Janeiro, Brazil: Phytogeographic considerations. Botanica Marina 35: 357-364.

Figueiredo, M.A.O. 1989. Ficoflora marinha bentônica do Município de Paraty, Rio de Janeiro. Universidade Federal do Rio de Janeiro, Rio de Janeiro. Dissertação de Mestrado.

Hoek, C. van den. 1982. A taxonomic revision of the American species of Cladophora (Chlorophyceae) in the North Atlantic Ocean and their geographic distribution. North Holland Publishing Company, Amsterdam.

Ikeda, Y. \& Stevenson, M. 1980. Determination of circulation and short period fluctuation in Ilha Grande Bay (RJ), Brazil. Boletim do Instituto Oceanográfico 29: 89-98.

Kanagawa, A.I. 1984. Clorofíceas bentônicas marinhas do Estado da Paraíba, Brasil. Parte I e II. Universidade de São Paulo, São Paulo. Tese de Doutorado.

Maciel, N.C.; Araújo, D.S.D. \& Magnanini, A. 1981. A situação ambiental dos ecossistemas da Praia do Sul e do leste, na Itha Grande, Município de Angra dos Reis, RJ, com vistas à preservação. FEEMA/DECAM. Rio de Janeiro. 29p.

Maciel, N.C.; Araújo, D.S.D. \& Magnanini, A. 1984. Reserva Biológica Estadual da Praia do Sul (Ilha Grande, Angra dos Reis, R.J.): Contribuição para o conhecimento da fauna e flora. Boletim Fundação Brasileira para Conservação da Natureza 19: 126-148

Pedrini, A.G. 1980. Algas marinhas bentônicas da Baía de Sepetiba e arredores (Rio de Janeiro). Universidade Federal do Rio de Janeiro, Rio de Janeiro. Dissertação de Mestrado.

Piqué, M.P.R. 1984. Estudos de Coralináceas articuladas (Rhodophyta) do litoral do Estado de São Paulo. Universidade Estadual Paulista, São Paulo. Dissertação de Mestrado.

Santos, D.P. 1983. Clorofíceas bentônicas do Estado de Santa Catarina. Universidade de São Paulo, São Paulo. Dissertação de Mestrado.

Signorini, S.R. 1980 a. A study of the circulation in Bay of Ilha Grande and Bay of Sepetiba. Part I. A survey of the circulation based on experimental field data. Boletim do Instituto Oceanográfico 29: 41-55

Signorini, S.R. 1980 b. A study of the circulation in Bay of Ilha Grande and Bay of Sepetiba. Part II. An assessment of the tidally and wind-driven circulation using a finite element numerical model. Boletim do Instituto Oceanográfico 29:57-68

Széchy, M.T.M. 1986. Feofíceas do litoral norte do Estado do Rio de Janeiro, Brasil. Universidade Federal do Rio de Janeiro, Rio de Janeiro. Dissertação de Mestrado.

Széchy, M.T.M. \& Cordeiro-Marino, M. 1991. Feofíceas do litoral norte do Estado do Rio de Janeiro, Brasil. Hoehnea 18(1): 205-241.

Wynne, M.J. 1986. A checklist of benthic marine algae of the tropical and subtropical western Atlantic. Canadian Journal of Botany 64: 2239-2281.

Yoneshigue, Y. 1985. Taxonomie et écologie des algues marines dans la région de Cabo Frio (Rio de Janeiro - Brésil). Faculté des Sciences de Luminy. France, Marseille. Tese de Doutorado.

Yoneshigue-Braga, Y. 1971. Flora marinha bentônica da Baía de Guanabara e cercanias III. Rhodophyta 1. Goniothricales, Bangiales, Compsogonales, Nemalionales, Gelidiales. Instituto de Pesquisas da Marinha 35: $1-36$.

Yoneshigue-Braga, Y. 1972a. Flora marinha bentônica da Baía de Guanabara e cercanias III. Rhodophyta 2. Cryptonemiales, Gigartinales e Rhodymeniales. Instituto de Pesquisas da Marinha 62: 1-39.

Yoneshigue-Braga, Y. 1972 b. Flora marinha bentônica da Baía de Guanabara e cercanias III. Rhodophyta 3. Ceramiales. Instituto de Pesquisas da Marinha 61: 1-49. 